\title{
Construction Features and Analisis of Warfar Information Model with Markov Switchings under Levy Approximation Conditions
}

\author{
Igor SAMOILENKO ${ }^{1}$, Anatolii NIKITIN²
}

${ }^{1}$ Department of Operations research, Faculty of Computer Science and Cybernetics, Taras Shevchenko National University of Kyiv,Glushkov av. 4D, UA-03680,Kyiv, Ukraine University,

${ }^{2}$ Department of Science, Faculty of Computer Science and Cybernetics, Taras Shevchenko National University of Kyiv,Glushkov av. 4D, UA-03680,Kyiv, Ukraine University,

E-mails: ${ }^{1}$ isamoil@i.ua; ${ }^{2}$ nikitin2505@univ.kiev.ua

\begin{abstract}
We construct and study a continuous model that describes the conflict interaction of two complex systems with non-trivial internal structures. External conflict interaction is modeled by the additional influence of chance. The dynamics of internal conflict are similar to the Lotka-Volterra model, namely the model of information warfare. We interpret the new model of information warfare as the influence of rare events that rapidly change certain ideas of a large number of people. As a result, the number of supporters of different ideas make stochastic jumps that we can see using the Levy approximation scheme. We suggest that such a model could be more natural, as important news now has a quick and wonderful impact on audiences through television and the Internet.
\end{abstract}

KEY WORDS: stochastic evolutionary system, information warfare model; limiting distributions, Lotka-Volterra equations

\section{Introduction}

The Lotka-Volterra model of prey-predator interaction is one of the main models for simulation of many processes in applied mathematics, social sciences and economics [3], [8-11]. Application of this approach to information warfare model was proposed in [4]. Authors regard some social community of quantity N0, potentially exposed some information threat (InfT) of two types, that is, for example, the threat of a negative change in its state by transmitting some information relevant to this group by information two different channels. The values $N 1(t), N 2(t)-$ the numbers of "adherents" depending on time t who accepted the new information, ideas, norms, etc. of the type 1 and 2 respectively. These are the main current characteristics of the degree of prevalence of InfT. We propose some results where the generator of the limit process is constructed in explicit form. We also give some interpretation of our model.

\section{Classical Information Warfare Model}

The main model assumptions are:

Both InfT are distributed among the community through the two information channels:

- the first one is "external" in relation to the community, for example, advertising media campaign. Its intensity is characterized by the parameters $\alpha 1>0$ and $\alpha 2>0$ respectively, both are considered to be independent of time;

- the second, "internal" channel is interpersonal communication between members of the social community (its intensity, that is, the number of equivalent informational contacts, characterized by the parameters $\beta 1$ $>0$ and $\beta 2>0$ respectively, that are also independent of time). As a result, the adherents of the first idea that have been already "recruited" (their number is equal to $\mathrm{N} 1(\mathrm{t})$ ), make their personal contribution to the recruitment process by affecting non-recruited members (their number is equal to the value of N0 $\mathrm{N} 1(\mathrm{t})-\mathrm{N} 2(\mathrm{t}))$. The same is for the adherents of the second idea.

1. The rate of change of the number of adherents $N_{1}(t)$ and $N_{2}(t)$ (that is, the number recruited into the unit time) consists of:

- external recruitment rate (it is proportional to the product of the intensities $\alpha 1$ and $\alpha 2$ and on the number of individuals who are not yet recruited N0 - N1(t) - N2(t)), that is, $\alpha 1$. $(\mathrm{N} 0-\mathrm{N} 1(\mathrm{t})-\mathrm{N} 2(\mathrm{t}))$ and $\alpha 2 \cdot(\mathrm{N} 0-\mathrm{N} 1(\mathrm{t})-\mathrm{N} 2(\mathrm{t}))$ respectively;

\footnotetext{
${ }^{1}$ Corresponding author.

E-mail address: isamoil@i.ua.
} 
- internal recruitment rate (it is proportional to the product of intensities $\beta 1$ and $\beta 2$, on the corresponding number of active adherents $N 1(t), N 2(t)$ and on the number nonrecruited $\mathrm{N} 0-\mathrm{N} 1(\mathrm{t})-\mathrm{N} 2(\mathrm{t}))$, that is, $\beta 1 \cdot \mathrm{N} 1(\mathrm{t})(\mathrm{N} 0-\mathrm{N} 1(\mathrm{t})-\mathrm{N} 2(\mathrm{t}))$ and $\beta 2 \cdot \mathrm{N} 2(\mathrm{t})(\mathrm{N} 0-\mathrm{N} 1(\mathrm{t})-\mathrm{N} 2(\mathrm{t}))$ respectively.

The model is, thus, described by Lotka-Volterra-type equations:

$$
\begin{gathered}
d N_{1}(t) / d t=\left(\alpha_{1}+\beta_{1} N_{1}(t)\right)\left(N_{0}-N_{1}(t)-N_{2}(t)\right), \\
d N_{2}(t) / d t=\left(\alpha_{2}+\beta_{2} N_{2}(t)\right)\left(N_{0}-N_{1}(t)-N_{2}(t)\right), t>0 .
\end{gathered}
$$

\section{Information Warfare Model with Impulsive Influence}

As we know, the outside world speaks with us the language of probability theory, so the deterministic model is only part of the real situation. That is why we are building a model that describes a model of information warfare that has not yet been explored, that is, a model based on contingencies, and contingencies of different types:

$$
d N^{\varepsilon}(t)=C\left(N^{\varepsilon}(t), x\left(t / \varepsilon^{2}\right)\right) d t+d \eta^{\varepsilon}(t)
$$

where

$$
C\left(N^{\varepsilon}(t), x\left(t / \varepsilon^{2}\right)\right)=\left(\begin{array}{cc}
-\alpha_{1}(x)+\beta_{1}(x) N_{0}(x)-\beta_{2}(x) N_{1}^{\varepsilon}(t) & -\alpha_{1}(x)-\beta_{1}(x) N_{1}^{\varepsilon}(t) \\
-\alpha_{2}(x)-\beta_{2}(x) N_{2}^{\varepsilon}(t) & -\alpha_{2}(x)+\beta_{2}(x) N_{0}(x)-\beta_{1}(x) N_{2}^{\varepsilon}(t)
\end{array}\right)\left(\begin{array}{l}
N_{1}^{\varepsilon}(t) \\
N_{2}^{\varepsilon}(t)
\end{array}\right)
$$

$\varepsilon$ is a small series parameter;

$N^{\varepsilon}(t)$ is a two-dimensional vector of solutions, components of which are the quantities of the adherents of different ideas;

$x\left(t / \varepsilon^{2}\right)$ is uniformly ergodic Markov process in a standart pgase space $(X, \mathbf{X})$, is defined by the generator [1], [2], $[6],[13]$

$$
\mathbf{Q} \varphi(x)=q(x) \int_{X} P(x, d y)[\varphi(y)-\varphi(x)]
$$

on the Banach space $B(X)$ of real-valued bounded functions $\varphi(x)$ with the supremum norm

$$
\|\varphi\|=\max _{x \in X}|\varphi(x)| \text {. }
$$

The stochastic kernel $P(x, B), x \in X, B \in \mathbf{X}$, uniformly ergodic embedded Markov chain

$$
x_{n}=x\left(\tau_{n}\right), n \geq 0,
$$

with stationary distribution $\rho(B), B \in \mathbf{X}$. Stationary distribution, $\pi(B), B \in \mathbf{X}$, of the Markov process

$x(t), t \geq 0$ is defined by the relation

$$
\pi(d x) q(x)=q \rho(d x)
$$

where

$$
q=\int_{X} \pi(d x) q(x)
$$

Denote by $R_{0}$ potential operator of the generator $\mathrm{Q}$, which is defined by the equality

$$
R_{0}=\Pi-(\Pi+\mathbf{Q})^{-1}
$$

where $\Pi \varphi(x)=\int_{X} \pi(d y) \varphi(y) \mathbf{1}(x)$ is the projector of zeroes of generator Q onto subspace

$$
N_{Q}=\{\varphi: \mathbf{Q} \varphi=0\}
$$

$\eta^{s}(t)$ - is the impulse perturbation process [1], [5], [6], [7], [16] in the Levy approximation scheme, defined by the relation 


$$
\eta^{\varepsilon}(\mathrm{t})=\int_{0}^{t} \eta^{\varepsilon}\left(d s, x\left(s / \varepsilon^{2}\right)\right),
$$

where the family of processes with independent increments

$$
\Gamma^{\varepsilon}(x) \varphi(\omega)=\varepsilon^{-2} \int_{R}(\varphi(\omega+\mathrm{v})-\varphi(\omega)) \Gamma^{\varepsilon}(d \mathrm{v}, x), x \in X
$$

and satisfies the properties of Lévy approximation;

L1. The approximation of averages

$$
\int_{R} \mathrm{v} \Gamma^{\varepsilon}(d \mathrm{v}, x)=\varepsilon a_{1}(x)+\varepsilon^{2}\left(a_{2}(x)+\theta_{a}(x)\right), \quad O_{a}(x) \rightarrow 0, \varepsilon \rightarrow 0,
$$

and

$$
\int_{R} \mathrm{v}^{2} \Gamma^{s}(d \mathrm{v}, x)=\varepsilon^{2}\left(b(x)+\theta_{b}(x)\right), \quad \theta_{b}(x) \rightarrow 0, \varepsilon \rightarrow 0 .
$$

L2. The condition imposed on the distribution function

$$
\int_{R} g(\mathrm{v}) \Gamma^{\varepsilon}(d \mathrm{v}, x)=\varepsilon^{2}\left(\Gamma_{g}(x)+\theta_{g}(x)\right), \theta_{g}(x) \rightarrow 0, \varepsilon \rightarrow 0
$$

for all $g(v) \in C_{3}(R)$, and $C_{3}(R) \quad$ is the space of real-valued bounded functions such that

$$
g(\mathrm{v}) /|\mathrm{v}|^{2} \rightarrow 0,|\mathrm{v}| \rightarrow 0
$$

where measure $\Gamma_{g}(\mathrm{x})$ is bounded for all $g(\mathrm{v}) \in C_{3}(R)$ and is defined by the relation (functions from the space $C_{3}(R)$ separate the measures):

$$
\Gamma_{g}(x)=\int_{n} g(v) \Gamma_{0}(d v, x), \quad g(v) \in C_{3}(R) .
$$

L3. The uniform quadratic integrability

\section{Asymptotic Analisys of the Model}

$$
\lim _{c \rightarrow \infty} \int_{|v|>c} \mathrm{v}^{2} \Gamma_{0}(d \mathrm{v}, x)=0
$$

The stochastic evolutionary system in an ergodic Markov medium is defined by the stochastic diffenential equation (1). Assume that the balance condition is fulfilled

$$
a_{1}:=\int_{-} \pi(d x) a_{1}(x)=0
$$

First, let us consider the asymptotic properties of the perturbation process.

Theorem 1. If balance condition and conditions $\mathbf{L} \mathbf{1}-\mathbf{L} 3$ are satisfied, weak convergence

$$
\eta^{s}(t) \rightarrow \eta^{0}(t), \varepsilon \rightarrow 0 .
$$

takes pllace for the the impulse perturbation process.

The limiting process $\eta^{0}(t)$ is defined by the generator

where

$$
\Gamma \varphi(w)=\hat{a}_{2} \varphi^{\prime}(w)+\frac{1}{2} \sigma^{2} \varphi^{\prime \prime}(w)+\int_{R}[\varphi(w+v)-\varphi(v)] \hat{\Gamma}_{0}(d v),
$$

$$
\begin{gathered}
\hat{a}_{2}=\int_{X} \pi(d x)\left(a_{2}(x)-a_{0}(x)\right), \\
\sigma^{2}=\int_{X} \pi(d x)\left(b(x)-b_{0}(x)\right)+2 \int_{X} \pi(d x) a_{1}(x) R_{0} a_{1}(x),
\end{gathered}
$$




$$
\begin{gathered}
a_{0}(x)=\int_{R} v \Gamma_{0}(d v, x), \\
b_{0}(x)=\int_{R} v^{2} \Gamma_{0}(d v, x), \\
\hat{\Gamma}_{0}(v)=\int_{v} \pi(d x) \Gamma_{0}(v, x) .
\end{gathered}
$$

Thus, we deal in a limit with Lévy process, which gas three components: derministic drift, diffusion component and Poisson jumping component.

Further we investigate the asymptotic properties of the original evolutionary system (1), in particular, using the approaches proposed in [14], [15].

Theorem 2. If the balance condition and conditions L1 - L3 are satisfied, the weak convergence in the sense of generators convergence

$$
\left(u^{s}(t), \eta^{s}(t)\right) \rightarrow\left(u^{0}(t), \eta^{0}(t)\right), \varepsilon \rightarrow 0
$$

takes place.

The limiting coupled process is defined by the generator

$$
\mathbf{L} \varphi(w, v)=\hat{C}(u) \varphi^{\prime}(w, \square)+\Gamma_{\omega} \varphi(w, \neg)+\Gamma_{v} \varphi(\sqcap, v)
$$

where the generators $\Gamma_{\mathrm{w}}$ and $\Gamma_{\mathrm{v}}$ are the same generators ( $\Gamma$, defined in Theorem 1), but acting on different arguments of the vector-function $\varphi(w, v)$, corresponding to a coupled process.

The averaged function

$$
\hat{C}(u)=\int_{X} \pi(d x) C(u, x)
$$

The last correlation means that to obtain the limit characteristics that describe information warfare model, all the functions in (2) that depend on x should be averaged by the stationary measure of the switching Markov process.

\section{Investigation Methods and Results}

We apply the approaches to the construction and analysis of complex systems proposed in the works of Koroluk V.S. [1], [2] and his followers, in particular, we apply the following scheme:

1. Construction of the generator of the Markov additive process.

2. Asymptotic form of the generator acting on some special type of test functions.

3. Solving of a singular perturbation problem on test functions in a form

$$
\varphi^{\varepsilon}(u, w, v)=\varphi(u, w)+\varepsilon \varphi_{1}(u, \dot{w}, v)+\varepsilon^{2} \varphi_{2}(u, w, v) .
$$

The following resumes should be made:

1. The weak convergence of the processes

$$
u^{\varepsilon}(t) \Rightarrow u^{0}(t), \varepsilon \rightarrow 0
$$

follows from the convergence of respective generators when compactness of prelimiting set of processes $u^{\varepsilon}(t)$ holds true. Weak convergence of stochastic processes is usually proved by checking the two conditions: tightness of the distributions of the converging processes which ensures the existence of a converging subsequence and uniqueness of the weak limit. The passage to the limit can be done on the semigroups which correspond to the converging processes as well as on appropriate generators. While proving convergence of generators a natural question arises concerning the uniqueness of a limit semigroup. It can be answered by representing the process in focus as a unique solution to a martingale problem which is formulated with the help of the limit generator.

1.The limit process $u^{0}(t)$ can be given by stochastic differential equation

where

$$
d \hat{u}(t)=\left[\hat{C}(\hat{u}(t))+\hat{a}_{2}\right] d t+\sigma d w(t)+\int_{R} v \tilde{v}(d t, d \mathrm{v})
$$

$$
\mathbf{E} \tilde{v}(d t, d v)=d t \tilde{\Gamma}_{0}(d v)
$$


The limit process $u^{0}(t)$ has three components. The deterministic drift is defined by the solution of the differential equation

$$
d \hat{u}_{d}(t)=\left[\hat{C}\left(\hat{u}_{d}(t)\right)+\hat{a}_{2}\right] d t
$$

where the additional term $\hat{a}_{2}$ appears due to accumulation with the normalized time $t / \varepsilon^{2}, \varepsilon \rightarrow 0$ of small jumps of the impulse process that happen with probability, close to one. The second, diffusion component is defined by a parameter $\sigma$ and it arises due to accumulation with growth of the normalized time $t / \varepsilon^{2}, \varepsilon \rightarrow 0$ of small jumps of degree $\varepsilon$, that happen with probability, close to one too. The third component is rare big jumps that take place with nearly zero probability and are defined in terms of averaged measure of jumps $\Gamma_{0}(\mathrm{du})$ by the generator

$$
\Gamma_{j} \varphi(w)=\int_{R}[\varphi(w+u,\urcorner-\varphi(u, \square)] \Gamma_{0}(d u)
$$

\section{Interpretation}

In many works on mathematical biology and economics the modelling of population dynamics or economical processes is based on Lotka-Volterra type equations. As a rule, deterministic continuous models are studied.

We propose a new model of information warfare with an additional influence of chance. That may be interpreted as some kind of rare events that rapidly change some beliefs of large quantities of people. As a result, the quantities of adherents of different ideas make stochastic jumps, that we may see applying Lévy approximation scheme. We suppose that such a model could be more natural, as soon as now breaking news produce quick and astonishing influence on the audience through TV and Internet.

The behavior of our model could not be analyzed obviously for any fixed moment of time as it was done in a classical case. But, as it is usual for stochastic models, we may obtain functional limit theorems that present the behavior on large time intervals. Thus, we obtain averaged limit characteristics of the process and may use them to construct obvious solutions. We hope to obtain recommendations for prevalence strategies in information warfare fights in future.

\section{Conclusions}

The following results of our investigation were obtained:

- a model that is more general then the classical one was proposed;

- the limit generator of the dynamical system was constructed;

- the behavior of the limit process in terms of its components was analyzed.

\section{References}

1. Korolyuk V.S. Stochastic Systems in Merging Phase Space / V.S. Korolyuk, N. Limnios // World Scientific, 2005. - $330 \mathrm{c}$.

2. Korolyuk V.S. Lévy and Poisson approximations of switched stochastic systems by a semimartingale approach / V.S. Korolyuk, N.Limnios, I.V. Samoilenko // Comptes Rendus Mathématique, 354, 2016, 723-728.

3. Lotka, A. J.: Relation between birth rates and death rates. Science. Vol. 26: 21-22 (1907). doi: 10.1126/ science.26.653.21-a

4. Mikhailov, A.P., Marevtseva, N.A. Models of information warfare. Math. Models Comput. Simul. Volume 4, Issue 3.: 251-259 (2012). doi: 10.1134/S2070048212030076

5. Samolilenko I.V. Differential Equations with Small Stochastic Terms Under the Lévy Approximating Conditions /I.V. Samoilenko, A.V. Nikitin // Ukrainian Mathematical Journal, 2018

6. Nikitin A.V. Asymptotic dissipativity of stochastic processes with impulsive perturbation in the levy approximation scheme / A.V. Nikitin // Journal of Automation and Information Sciences (2018)

7. Chabanyuk Y.M. Asymptotic properties of the impulse perturbation process under Levy approximation conditions with the point of equilibrium of the quality criterion / Y.M. Chabanyuk,, A.V. Nikitin, , U.T. Khimka // Matematychni Studii (2019)

8. Stone, L., Olinky, R. Phenomena in ecological systems, Experimental Chaos: 6th Experimental Chaos Conference, pp. 476-487 (2003)

9. Takahashi, K. I., Salam, K. Md. M. Mathematical model of conflict with non-annihilating multi-opponent. J. Interdisciplinary Math. Vol. 9 (3): 459-473 (2006). doi: 10.1080/ 09720502.2006.10700457

10. Tufto, J.: Effects of releasing maladapted individuals: a demographic evolutionary model. The American 
Naturalist. Vol. 158 (4): 331-340 (2001). doi: 10.1086/321987

11. Verhulst, P. P. Notice sur la loi que la population suit dans son accroissement. Correspondence mathematique et physique publiee par A. Quetelet. Vol. 10: 113-121 (1838)

12. Volterra, V. Sui tentativi di applicazione della matematiche alle scienze biologiche e sociali. Giornale degli Economisti. Vol. 23: 436-458 (1901).

13. Nikitin A.V. Asymptotic Properties of a Stochastic Diffusion Transfer Process with an Equilibrium Point of a Quality Criterion / A.V. Nikitin // Cybernetics and System analysis. - 2015. - Volume 51, Issue 4. - P. 650-656.

14. Nevelson M. B. Stochastic approximation and recursive estimation / Nevelson M. B., Khas'minskii, R. Z. // Moscow: Nauka, 1972. - 298 p.

15. Papanicolaou G., Stroock D., Varadhan S.R.S. Martingale approach to some limit theorems, Duke turbulence conference (Durham, NC, April 23-25, 1976), Duke University Mathematics Series III, New York: Duke University, 1977, $120 \mathrm{p}$.

16. Samoilenko I.V. Differential Equations with Small Stochastic Additions Under Poisson Approximation Conditions / I.V.Samoilenko, Y.M. Chabanyuk, A.V. Nikitin, U.T. Khimka // Cybernetics and System analysis. 2017. - Volume 53, Issue 3-P.410-416. 